\title{
Direct Transfer of Metallic Photonic Structures Onto End Facets of Optical Fibers
}

\author{
Xinping Zhang *, Feifei Liu and Yuanhai Lin \\ Institute of Information Photonics Technology and College of Applied Sciences, Beijing University of Technology, Beijing, China
}

We present a flexible approach to transfer metallic photonic crystals (MPCs) onto end facets of optical fibers. The MPCs were initially fabricated on a glass substrate with a spacer layer of indium tin oxide (ITO), which was used as a buffer layer in the transferring process. The fiber ends were firstly welded on the top surface of the MPCs by a drop of polymer solution after the solvent evaporated. The ITO layer was then etched by hydrochloric acid $(\mathrm{HCl})$, so that the MPCs got off the substrate and were transferred to the fiber ends. Alternatively, the MPCs may be also etched off the substrate first by immersing the sample in $\mathrm{HCl}$. The ultra-thin MPC sheet consisting of gold nanolines interlaced with photoresist gratings was then transferred to cap the fiber ends. In the later approach, we can choose which side of the MPCs to be used

OPEN ACCESS

Edited by:

Yanpeng Zhang,

Xi'an Jiaotong University, China

Reviewed by:

Feng $L i$,

University of Sheffield, UK Lei Zhang,

National University of Singapore,

Singapore

*Correspondence:

Xinping Zhang

zhangxinping@bjut.edu.cn

Specialty section:

This article was submitted to

Optics and Photonics,

a section of the journal

Frontiers in Physics

Received: 13 June 2016

Accepted: 08 July 2016

Published: 26 July 2016

Citation:

Zhang XP, Liu FF and Lin YH (2016)

Direct Transfer of Metallic Photonic

Structures Onto End Facets of Optical

Fibers. Front. Phys. 4:31.

doi: 10.3389/fphy.2016.00031 as the contact with the fiber facet. Such methods enabled convenient nano-structuring on optical fiber tips and achieving miniaturized MPC devices with compact integration, extending significantly applications of MPCs. In particular, the fabrications presented in this manuscript enrich the lab-on-fiber engineering techniques and the resultant devices have potential applications in remote sensing and detection systems.

Keywords: direct transfer, metallic photonic crystals, optical fibers, end facets, welding transfer, lift-off transfer

\section{INTRODUCTION}

Integration of photonic structures onto the end facets of optical fibers attracted extensive research interests for achieving miniaturized devices of multifold functions [1-6]. A variety of techniques have been reported for direct nano-structuring $[7,8]$ or indirect photonic nano-transferring on the fiber ends $[9,10]$. However, due to the small area of the end facet of an optical fiber and difficulties in managing flexible fibers in conventional nano-fabrication systems, multiple challenges need to be overcome in direct approaches. In contrast, it is much more convenient to fabricate highquality large-area photonic structures on planar substrates than on fiber ends, therefore, the indirect techniques generally facilitate more flexible and more promising device engineering. Furthermore, the indirect methods also lower the requirements on the materials for nano-structuring. In this case, all of the challenges are moved to the transferring techniques. Nanoimprinting lithography $[11,12]$ and capping with mechanical contact $[13,14]$ are some typical methods for fiber-end-based nanotransferring techniques. We have also demonstrated nano-structuring techniques for sensing and lasing devices $[15,16]$.

Here we can define direct and indirect transfer methods, where direct transfer means that the transferred photonic structures are the same as those initially fabricated, however, in the indirect processes the transferred structures are different from the precursor or the template in materials or 
in the reversal configuration. Thus, nanoimprinting transfer [10] or masked etching transfer [17] are indirect techniques. In this work, we demonstrate practical techniques for direct transferring the precursor metallic photonic crystals (MPCs) originally fabricated on planar glass substrate to end facets of optical fibers. A buffer layer of indium tin oxide (ITO) is crucial for these nano-transferring techniques, which not only favors high-quality nano-fabrication and nano-characterization, but also enables easy lift-off between the photonic structures and the substrate due to its easy dissolution in hydrochloric acid. In particular, this technique is preferable for batch fabrication with high success rate.

\section{FABRICATION OF METALLIC PHOTONIC STRUCTURES}

Figures 1A-C show schematically the fabrication procedures of the MPCs using interference lithography and colloidal gold nanoparticles, as has been described in our previous publications [18-20]. Interference lithography using a UV laser at $325 \mathrm{~nm}$ and a positive photoresist (PR) S1805 produced grating structures with a period of about $425 \mathrm{~nm}$ and a modulation depth of more than $100 \mathrm{~nm}$. Colloidal gold nanoparticles with a concentration of $100 \mathrm{mg} / \mathrm{ml}$ in xylene was spin-coated onto the PR grating. After the sample was heated to about $350^{\circ} \mathrm{C}$ for about $20 \mathrm{~min}$, metallic gratings consisting of gold nanolines interlaced with PR lines were produced. Figure 1D shows the SEM image of the fabricated gold nanoline gratings, which is called as one-dimensional MPCs and is used as the precursor for the subsequent transfer procedures.

\section{"WELDING" TRANSFER}

The so-called "welding transfer" refers to the process fixing the free end of an optical fiber to the MPCs with the facet of the fiber parallel to the plane of the MPC substrate. The "solder" here is the solution of polymethyl methacrylate (PMMA) in xylene with a concentration of $5 \mathrm{mg} / \mathrm{ml}$. In the process of welding transfer, an optical fiber was mounted such that the axis of the fiber is perpendicular to the plane of the above fabricated MPCs with end facet of the fiber touch the top surface of the MPCs, as shown in Figure 2A. Then, a drop of PMMA solution was added to the touching point between the fiber and the MPCs to fill up the gap between them. The free fiber end was fixed firmly onto the MPCs by PMMA after the solvent evaporates completely.

The welded sample was then immersed in hydrochloric acid with a concentration of $20 \%$ for about $30 \mathrm{~min}$, as shown in Figure 2B. After the ITO layer was dissolved into $\mathrm{HCl}$ thoroughly, the MPC structures were thus transferred completely onto the end facet of the optical fiber. The sample were rinsed in water and then trimmed to finish the transfer process, as shown in Figure 2C.

Figure 3 gives a clearer demonstration of the practical fabrication technique. Figures 3A,B show how the MPCs on a single piece of substrate can be transferred to a number of fiber ends. The process in Figure $\mathbf{3 A}$ is defined as a batch transfer, whereas, that in Figure 3B is defined as discrete transfer, where the MPC layer was firstly divided before the welding process. Figure 3C shows a finished sample, where the MPC device has been transferred and fixed onto one end of an optical fiber. The fiber has core diameter of 400 microns.

\section{LIFT-OFF TRANSFER}

In the lift-off transfer, the MPCs fabricated using a photoresist master grating on a ITO glass substrate was first immerse in $\mathrm{HCl}$ with a concentration of $20 \%$ for more than $30 \mathrm{~min}$. The MPCs interlaced with the PR grating was removed completely from the substrate after the ITO buffer layer was dissolved into $\mathrm{HCl}$. The MPC film was then floating on the top of the liquid, as shown in Figure 4A. The lift-off MPCs was picked up using a plastic tweezer, and pulled slowly on the surface of water with either side rinsed by water, as shown in Figure 4B. The photoresist with enhanced rigidity allows us to perform the rinsing process. A special holder was designed to pick up the lift-off MPC film from water. The MPC film was then placed on the fiber end, as shown in Figure 4C. In the final stage, the MPC film was trimmed to finish the transfer procedure after the holder was removed, as shown in Figure 4D.

Figure 5 shows the photograph of a finished sample by transferring a MPC device to a fiber end with the enlarged facet image under the optical microscope shown in the right panel. We can observe strong light diffraction by the MPC at the fiber end. Clearly, the area of the MPCs on the fiber facet is homogeneous, indicating excellent contact, and successful transfer process.

\section{OPTICAL PERFORMANCE OF THE TRANSFERRED MPCs}

The optical performance of the transferred MPCs on a fiber end facet was measured using transmission optical extinction spectroscopy. Broadband light from a halogen lamp was sent into the incident end of the fiber. The output at the MPC-structured end was sent to a spectrometer after passing through a polarizer sheet. TE polarization is parallel to the gold nanolines in the MPC grating, whereas, TM polarization is perpendicular to the gold nanolines. Clearly, localized surface plasmon resonance can be excited in MPCs for TM polarization. In the transferring process, PMMA has filled the gap between the fiber end and the MPCs, there exists a thin layer of PMMA between the end facet of the fiber and the MPCs. Therefore, waveguide resonance mode may be excited in the thin layer of PMMA. As has been investigated extensively, [21-23]. Fano coupling between waveguide resonance mode and localized surface plasmon resonance can be observed in such MPC structures. Figure 6 shows the optical extinction measurement results, where the red and black curves show the measurement results for TE and TM polarization, respectively. A strong peak at about $650 \mathrm{~nm}$ with a bandwidth larger than $100 \mathrm{~nm}$ at FWHM may be observed for TE polarization, which resulted from the waveguide resonance mode. We have verified the waveguide-resonance-mode feature of this optical extinction peak by measuring the spectrum at an 

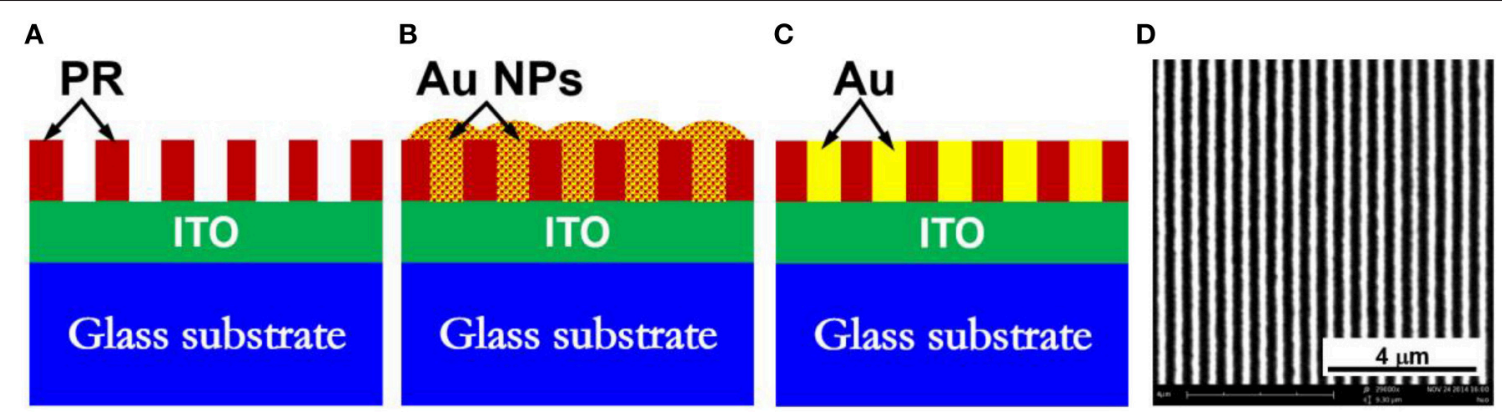

FIGURE 1 | Fabrication of MPCs using solution processed gold nanoparticles. (A) The photoresist (PR) grating fabricated using interference lithography. (B) Spin-coating of colloidal gold nanoparticles. (C) The MPCs consisting of gold nanolines interlaced with the PR grating after the annealing process. (D) The SEM image of the MPCs.
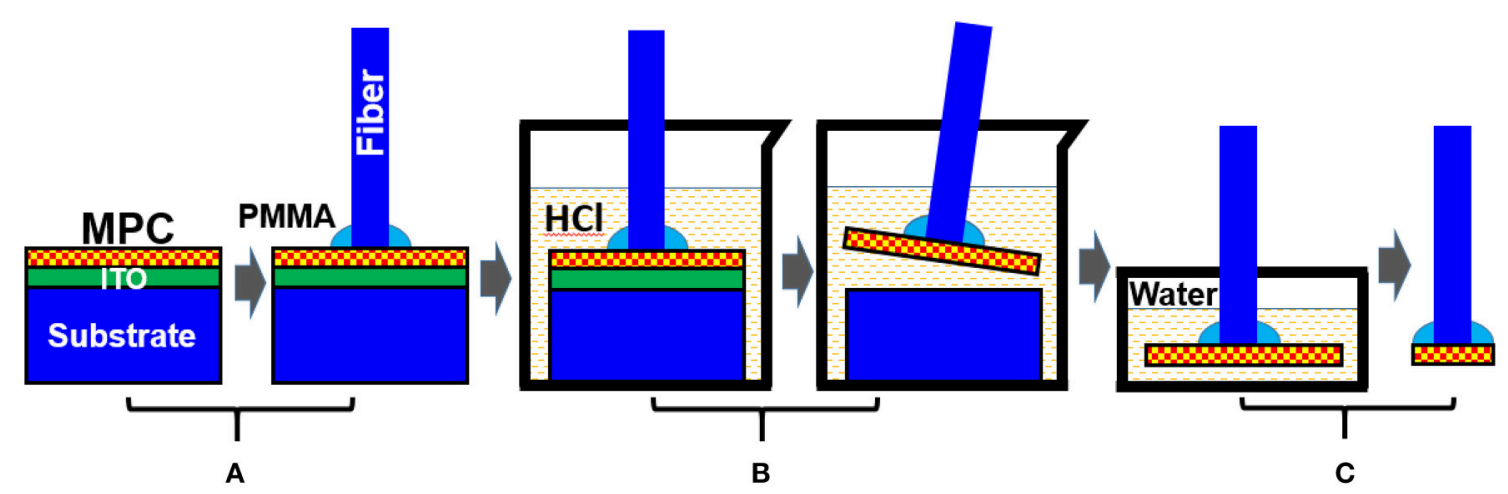

FIGURE 2 | "Welding" transfer process. (A) Welding of the fiber end to the MPCs using PMMA. (B) Etching of the ITO buffer layer. (C) Rinsing in water and trimming to finish the transfer.
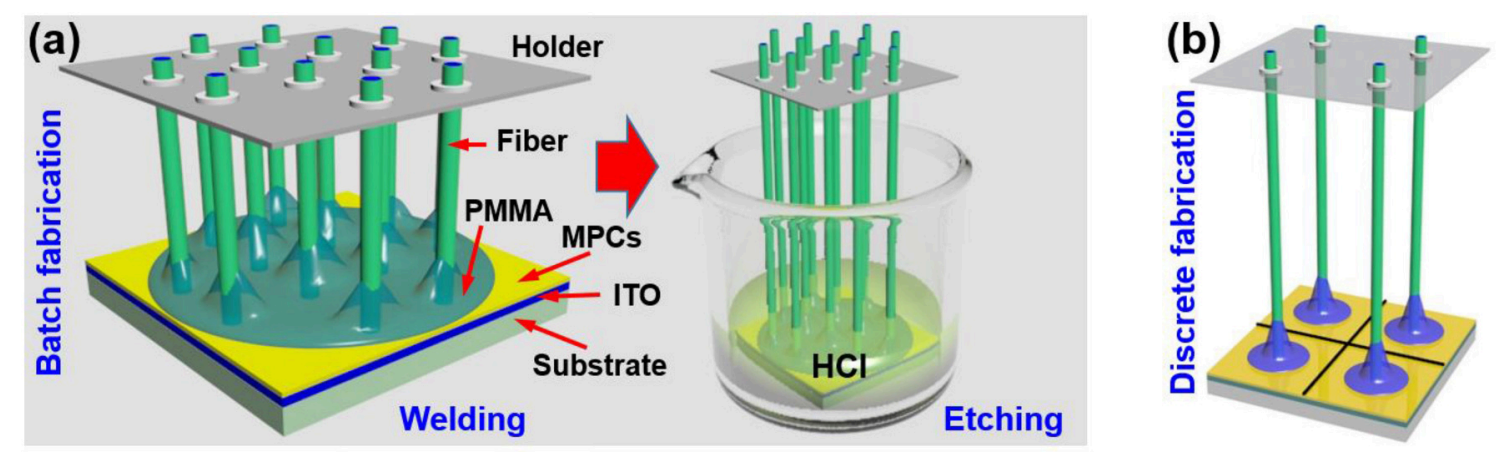

(c)

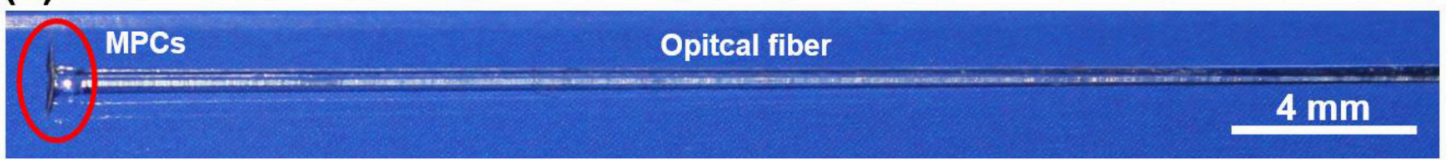

FIGURE 3 | (A,B) Schematic illustration of batch and discrete transfer of MPCs to the end facets of optical fibers, respectively. (C) A practical example of a transferred MPC device.

angle from the normal of the fiber facet. We observed splitting of this mode into two peaks, which is a typical feature of the waveguide resonance mode [24]. Large acceptance range of the incident angles in the fiber led to the broadband resonance mode. However, a dip centered at about $750 \mathrm{~nm}$ may be observed in the red curve for TM polarization, as indicated by a downward 


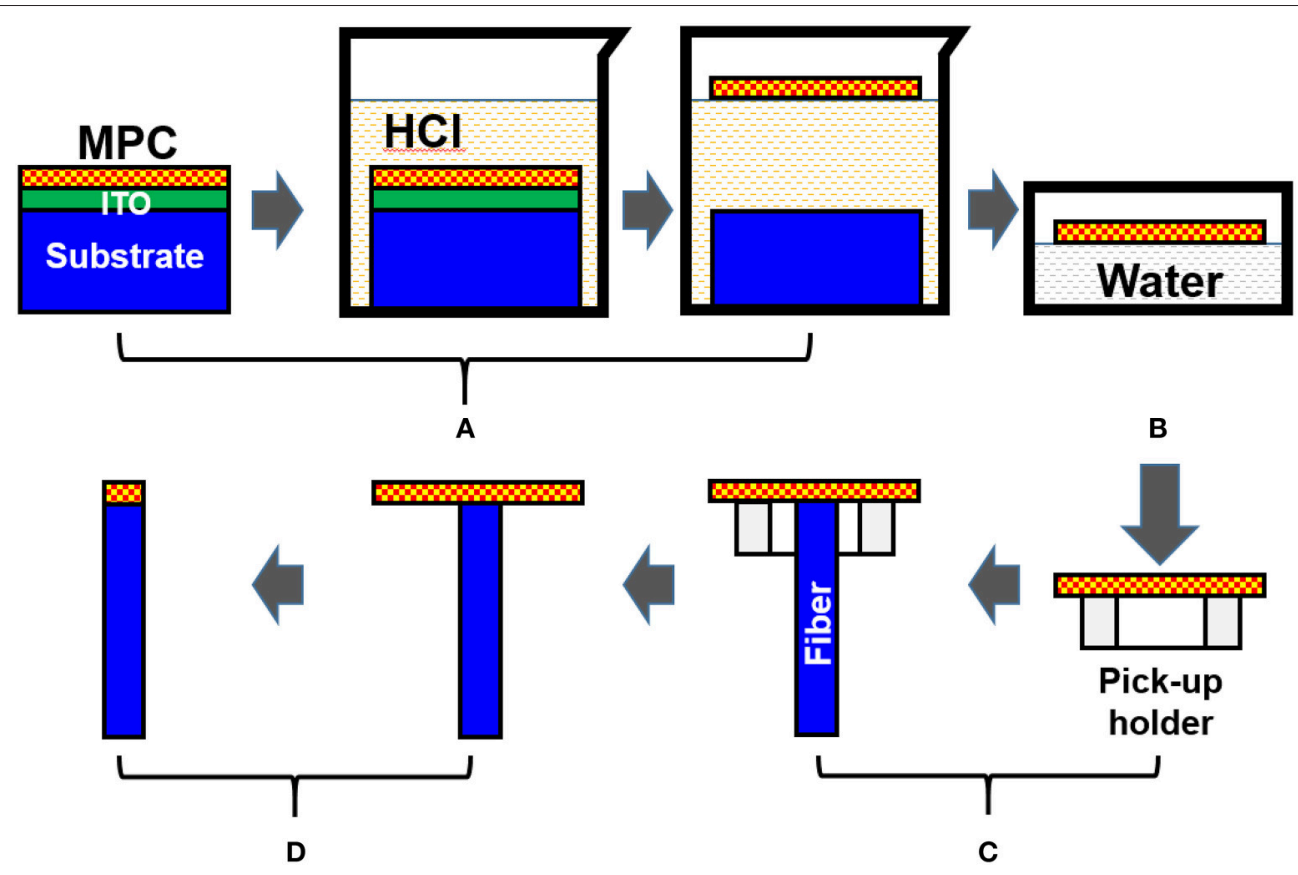

FIGURE 4 | Lift-off transfer processes. (A) Etching of the ITO buffer layer. (B) Rinsing in water. (C) Pick-up of the MPC film using a specially designed holder. (D) Trim the MPC film to finish the transfer.

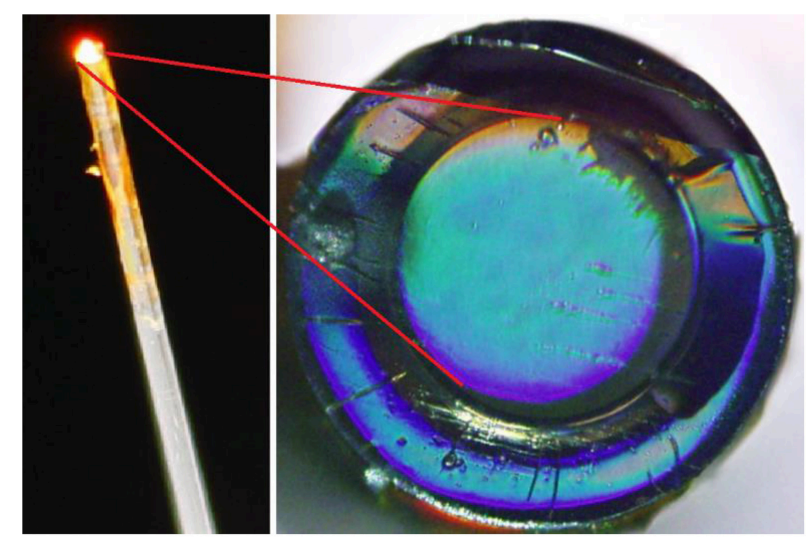

FIGURE 5 | The finished transfer of the MPCs to the end facet of an optical fiber using lift-off transfer with the optical microscopic observation of the MPCs on the fiber end.

red arrow, leaving two peaks on both sides. Improvements are still required to narrow the resonance spectrum, so that high sensitivity may be achieved for potential sensors applications based on the fiber-based MPCs.

\section{CONCLUSIONS}

We demonstrated multiple methods for direct transfer of MPCs to end facets of optical fibers. High efficiency, high flexibility, and high simplicity are the main advantages of these methods. Such kinds of integration of MPCs onto fibers enable a variety of

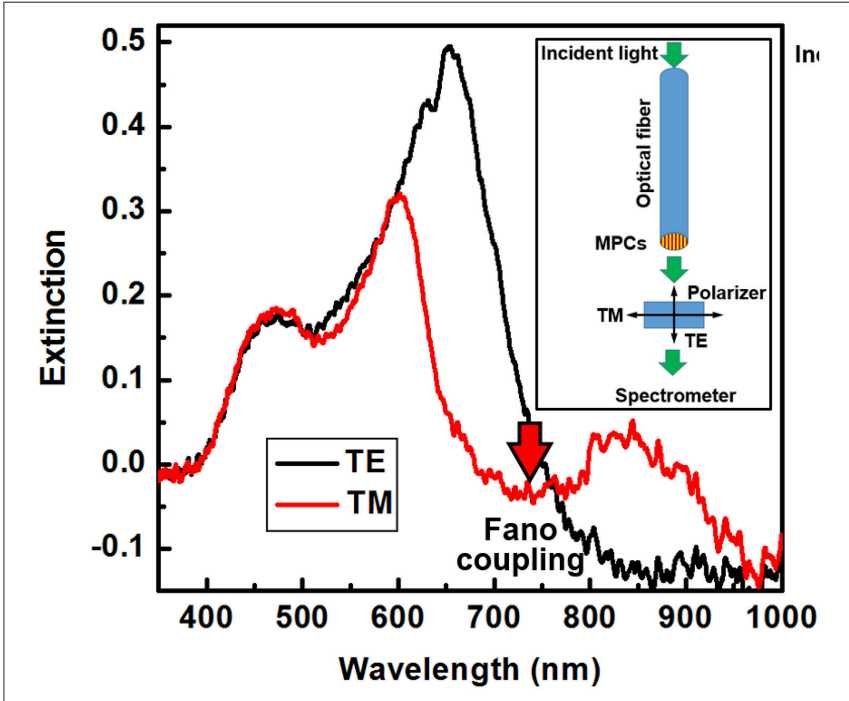

FIGURE 6 | Optical extinction spectroscopic measurements for different polarizations. The measurement setup is depicted schematically in the inset.

applications of plasmonic devices in remote sensors for extensive purposes, in particular for vibrational or acoustic sensors, and in compact optical instruments. The transferring methods lay basis for exploring new techniques for achieving miniaturized photonic devices. It needs to be stressed that for such MPC devices integrated to a fibers, it is suitable to develop sensor needles. 


\section{AUTHOR CONTRIBUTIONS}

$\mathrm{XZ}$ designed this research work, performed the fabrication and processed the data, and wrote the paper. FL measured the microscopic and spectroscopic data. YL provided the 3D pictures illustrating the transferring principles.

\section{REFERENCES}

1. Chen W, Han W, Abeysinghe DC, Nelson RL, Zhan Q. Generating cylindrical vector beams with subwavelength concentric metallic gratings fabricated on optical fibers. J Opt. (2011) 13:015003. doi: 10.1088/2040-8978/13/1/015003

2. Ma N, Ashok PC, Stevenson DJ, Gunn-Moore FJ, Dholakia K. Integrated optical transfection system using a microlens fiber combined with microfluidic gene delivery. Biomed Opt Express (2010) 1:694-705. doi: 10.1364/BOE.1.000694

3. Bachelot R, Fares A, Fikri R, Barchiesi D, Lerondel G, Royer P. Coupling semiconductor lasers into single-mode optical fibers by use of tips grown by photopolymerization. Opt Lett. (2004) 29:1971-3. doi: 10.1364/OL.29.001971

4. Parkes W, Djakov V, Barton JS, Watson S, MacPherson WN, Stevenson JTM, et al. Design and fabrication of dielectric diaphragm pressure sensors for applications to shock wave measurement in air. J Micromech Microeng. (2007) 17:1334-42. doi: 10.1088/0960-1317/17/7/016

5. Kostovski G, White DJ, Mitchell A, Austin MW, Stoddart PR. Nanoimprinted optical fibres: biotemplated nanostructures for SERS sensing. Biosens Bioelectron. (2009) 24:1531-5. doi: 10.1016/j.bios.2008.10.016

6. Viets C, Hill W. Comparison of fibre-optic SERS sensors with differently prepared tips. Sens Actuat B Chem. (1998) 51:92-9. doi: 10.1016/S09254005(98)00170-1

7. Williams HE, Freppon DJ, Kuebler SM, Rumpf RC, Melino MA. Fabrication of three-dimensional micro-photonic structures on the tip of optical fibers using SU-8. Opt Express (2011) 19:22910-22. doi: 10.1364/OE.19.022910

8. Feng S, Zhang X, Wang H, Xin M, Lu Z. Fiber coupled waveguide grating structures. Appl Phys Lett. (2010) 96:133101. doi: 10.1063/1.3373422

9. Smythe EJ, Dickey MD, Whitesides GM, Capasso F. A technique to transfer metallic nanoscale patterns to small and non-planar surfaces. ACS Nano (2009) 3:59-65. doi: 10.1021/nn800720r

10. Kostovski G, Chinnasamy U, Jayawardhana S, Stoddart PR, Mitchell A. Sub-15nm optical fiber nanoimprint lithography: a parallel, selfaligned and portable approach. Adv Mater. (2011) 23:531-5. doi: 10.1002/adma.201002796

11. Jia PP, Yang J. A plasmonic optical fiber patterned by template transfer as a high-performance flexible nanoprobe for real-time biosensing. Nanoscale (2014) 6:8836-43. doi: 10.1039/C4NR01411A

12. Scheerlinck S, Taillaert D, Thourhout DV, Baets R. Flexible metal grating based optical fiber probe for photonic integrated circuits. Appl Phys Lett. (2008) 92:031104. doi: 10.1063/1.2827589

13. Lipomi DJ, Martinez RV, Kats MA, Kang SH, Kim P, Aizenberg J, et al. Patterning the tips of optical fibers with metallic nanostructures using nanoskiving. Nano Lett. (2011) 11:632-6. doi: 10.1021/nl103730g

14. Wang B, Siahaan T, Dündar MA, Nötzel R, van der Hoek MJ, He $\mathrm{S}$, et al. Photonic crystal cavity on optical fiber facet for refractive

\section{ACKNOWLEDGMENTS}

The authors acknowledge the 973 program (2013CB922404), the National Natural Science Foundation of China (11274031, 11434016), and the Beijing Key Lab of Microstructure and Property of Advanced Materials for the support.

index sensing. Opt Lett. (2012) 37:833-5. doi: 10.1364/OL.37. 000833

15. Feng SF, Darmawi S, Henning T, Klar PJ, Zhang XP. A miniaturized sensor consisting of concentric metallic nanorings on the end facet of an optical fiber. Small (2012) 8:1937-44. doi: 10.1002/smll.201102290

16. Li ST, Wang L, Zhai TR, Xu ZY, Wang YL, Wang JY, et al. Plasmonic random laser on the fiber facet. Opt Express (2015) 23:23985-91. doi: 10.1364/OE.23.023985

17. Smythe EJ, Dickey MD, Bao J, Whitesides GM, Capasso F. Optical antenna arrays on a fiber facet for in situ surface-enhanced Raman scattering detection. Nano Lett. (2009) 9:1132-8. doi: 10.1021/nl803668u

18. Zhang XP, Liu HM, Feng SF. Solution-processible fabrication of largearea patterned and unpatterned gold nanostructures. Nanotechnology (2009) 20:425303. doi: 10.1088/0957-4484/20/42/425303

19. Zhang XP, Sun BQ, Guo HC, Tetreault N, Giessen H, Friend RH. Largearea two-dimensional photonic crystals of metallic nanocylinders based on colloidal gold nanoparticles. Appl Phys Lett. (2007) 90:133114. doi: $10.1063 / 1.2717567$

20. Zhang XP, Sun BQ, Guo HC, Nau D, Giessen H, Friend RH. Metallic photonic crystals based on solution-processible gold nanoparticles. Nano Lett. (2006) 6:651-5. doi: 10.1021/nl052361o

21. Christ A, Tikhodeev SG, Gippius NA, Kuhl J, Giessen H. Waveguideplasmon polaritons: strong coupling of photonic and electronic resonances in a metallic photonic crystal slab. Phys Rev Lett. (2003) 91:1839011. doi: 10.1103/PhysRevLett.91.183901

22. Zhang XP, Ma XM, Dou F, Zhao PX, Liu HM. A biosensor based on metallic photonic crystals for the detection of specific bioreactions. Adv Funct Mater. (2011) 21:4219-27. doi: 10.1002/adfm.201101366

23. Zhang XP, Dou F, Liu H. Molecular concentration sensor based on the diffraction resonance mode of gold nanowire gratings. Nanotechnology (2010) 21:335501. doi: 10.1088/0957-4484/21/33/335501

24. Rosenblatt D, Sharon A, Friesem AA. Resonant grating waveguide structures. IEEE J Quantum Electron. (1997) 33:2038-59. doi: 10.1109/3. 641320

Conflict of Interest Statement: The authors declare that the research was conducted in the absence of any commercial or financial relationships that could be construed as a potential conflict of interest.

Copyright (c) 2016 Zhang, Liu and Lin. This is an open-access article distributed under the terms of the Creative Commons Attribution License (CC BY). The use, distribution or reproduction in other forums is permitted, provided the original author(s) or licensor are credited and that the original publication in this journal is cited, in accordance with accepted academic practice. No use, distribution or reproduction is permitted which does not comply with these terms. 